\title{
DIFERENTES COMPOSIÇÕES DE SUBSTRATOS E AMBIENTES PROTEGIDOS NA FORMAÇÃO DE MUDAS DE PÉ-FRANCO DE TAMARINDEIRO ${ }^{1}$
}

\author{
EDILSON COSTA ${ }^{2}$, ANTONIO FLÁVIO ARRUDA FERREIRA ${ }^{3}$, \\ PRISCILLA NÁTALY DE LIMA SILVA ${ }^{3}$, EVANDRO MICHEL VALERO NARDELLI ${ }^{3}$
}

RESUMO-O tamarindeiro é uma frutífera importante ocorrente no Cerrado brasileiro e com sistema radicular profundo e possui resistência à seca prolongada. Seus frutos são utilizados na culinária; suas sementes, em forragem animal, e seu óleo, na indústria farmacêutica. Diante do exposto, este trabalho teve o objetivo de avaliar a formação de mudas de pé-franco de tamarindeiro, em diferentes composições de substratos e ambientes protegidos. As mudas foram produzidas em estufa agrícola, coberta com filme de polietileno de baixa densidade de $150 \mu \mathrm{m}$ e tela termorrefletora de $50 \%$ de sombreamento sob o filme; viveiro com tela preta de monofilamento de $50 \%$ de sombreamento e viveiro com tela termorrefletora de $50 \%$ de sombreamento. Em cada ambiente de cultivo, foram testados os substratos: $100 \%$ de solo; $80 \%$ de solo e $20 \%$ de composto orgânico; $60 \%$ de solo e $40 \%$ de composto orgânico; $40 \%$ de solo e $60 \%$ de composto orgânico; $20 \%$ de solo e $80 \%$ de composto orgânico, e $100 \%$ de composto orgânico. O efeito do ambiente foi examinado usando procedimento estatístico para análise de experimentos combinados (grupos de experimentos). Dentro dos ambientes, um delineamento experimental inteiramente casualizado foi adotado, com oito repetições. O substrato com $80 \%$ de solo e $20 \%$ de Organosuper ${ }^{\circledR}$ foi o mais propício para a formação das mudas. A estufa agrícola foi o melhor ambiente para a formação das mudas de tamarindeiro.

Termos para indexação: Tamarindus indica, Cerrado, Composto Orgânico, Estufa Agrícola, Telas de Sombreamento.

\section{SUBSTRATES COMPOSITION AND PROTECTED ENVIRONMENTS FOR TAMARIND SEEDLINGS}

\begin{abstract}
The tamarind is an important fruit occurring in the Brazilian Cerrado and with a deep root system having resistance to prolonged droughts. Its fruits are used in cooking, their seeds are used for animal fodder and its oil is used in the pharmaceutical companies. Given the above, this study had the aim to evaluable tamarind ungrafted seedlings formation in different substrates composition and protected environments. The seedlings were grown in greenhouse covered with low density polyethylene film of 150 micron and screen thermal-reflector of 50\% shading under film; nursery with black monofilament screen $50 \%$ of shading and nursery with screen thermal-reflector of $50 \%$ shading. In each cultivation environment, substrates were tested: $100 \%$ soil, $80 \%$ soil and $20 \%$ organic compost, $60 \%$ soil and $40 \%$ organic compost, $40 \%$ soil and $60 \%$ organic compost, $20 \%$ soil and $80 \%$ organic compost, and $100 \%$ organic compost. The effect of environment was examined using statistical procedures for analysis of combined experiments (experiments groups). Within environments a completely randomized design with eight replications. The substrate with $80 \%$ soil and $20 \%$ Organosuper ${ }^{\circledR}$ was the most conducive for seedlings. The greenhouse was the best environment for tamarind seedlings.
\end{abstract}

Index terms: Tamarindus indica, Cerrado, Organic Compound, Greenhouse, Screen of Shading.

(Trabalho 202-11). Recebido em: 08-08-2011. Aceito para publicação em: 05-09-2012.

${ }^{2}$ Professor Doutor da Universidade Estadual de Mato Grosso do Sul (UEMS), Unidade de Cassilândia. Rodovia MS 306, km 6,5. assilândia - MS. E-mail: mestrine@uems.br

${ }^{3}$ Graduandos (as) da UEMS, Bolsistas PIBIC, Unidade de Aquidauana. E-mails: tonho_flavio@yahoo.com.br; priscilla_nataly@ hotmail.com, evandro_nardelli@hotmail.com 


\section{INTRODUÇÃO}

A região de transição dos biomas Cerrado e Pantanal, no Estado de Mato Grosso do Sul, apresenta a ocorrência de diversas frutíferas, exóticas e nativas, com potencial promissor para o cultivo comercial. As ocupações destes biomas com a ampliação das fronteiras agrícolas de culturas anuais e o extrativismo predatório estão ameaçando a variabilidade dessas espécies. Estudos com exploração econômica de forma sustentável, através de plantios planejados, além de gerar emprego e renda, seriam uma forma de garantir a perpetuação dessas espécies.

$\mathrm{O}$ tamarindeiro, Tamarindus indica L., é uma frutífera importante ocorrente no Cerrado brasileiro, encontrada em muitos países da Ásia, África e América do Sul. Por apresentar sistema radicular profundo, possui resistência a secas prolongadas e, desta forma, é indicada em cultivo de regiões semiáridas. Sua utilização se dá, principalmente na culinária regional, bem como suas sementes servem como forragem animal, e o óleo extraído de suas sementes é de uso industrial. Segundo Kothari e Seshadri (2010), a acetona e o metanol extraídos de sementes de $T$. indica são ativos contra organismos gram-positivos e gram-negativos, além de o extrato metanólico possuir efeito bactericida. A casca do fruto do tamarindo apresenta elevada capacidade de adsorção, sendo usada como remoção de metal pesado (íons cromo) (RAO POPURI et al.2007). Na região de transição entre o Cerrado e o Pantanal, esta espécie é muito utilizada pelas comunidades locais; no entanto, existem escassos trabalhos sobre esta frutífera na região, provavelmente por ser pouco conhecida pelos brasileiros, exceção à região Nordeste.

É uma cultura perene, e o processo de produção das mudas deve garantir qualidade às plantas em exploração comercial. A qualidade da semente, do substrato e do adubo utilizados são fatores que afetam a produção de mudas de boa qualidade, pois contribuem para o melhor desenvolvimento e a sanidade da muda (YAMANISHI et al., 2004). Em tamarindeiro, Almeida et al. (2010) verificaram que sementes de maior massa proporcionam melhor desenvolvimento das mudas e não verificaram diferenças em qualidade, em função da profundidade de semeadura de 1;2 e $3 \mathrm{~cm}$. Para estabelecimento de sistemas agroflorestais, Flores et al. (2009) recomendam a formação de mudas com sementes recém- colhidas ou com, no máximo, um mês de armazenamento, com o hilo voltado para baixo.

Aliada a estes fatores, a utilização de ambientes protegidos pode promover maior uniformização das mudas formadas e obtenção de pomares homogêneos, sendo fundamental durante o processo de produção. A ambiência vegetal é o conjunto de elementos que compõem as condições climáticas da área de produção e visa a identificar as melhores condições micro-climáticas para o desenvolvimento das plantas e a obter melhores rendimentos e produtividades. Desta forma, existe grande importância no estudo da produção em ambientes protegidos, como estufas agrícolas, viveiro de mudas e casas de vegetação climatizadas, assim como no transporte de hortaliças e frutas, seu armazenamento e conservação.

Pesquisas com produção de mudas de frutíferas com substratos utilizando o composto orgânico Organosuper ${ }^{\circledR}$ e solo foram realizadas por Santos et al. (2011) com jatobá-do-cerrado, Costa et al. (2010 a) com maracujazeiro e Costa et al. (2011) com o mamoeiro. Estes autores verificaram que a quantidade do composto utilizada interage com o tipo de cobertura do ambiente protegido, sendo que Costa et al. $(2010$ a) destacam que doses de até $21 \%$ podem ser utilizadas na produção de mudas de maracujá em viveiro telado. Outros trabalhos utilizando este composto orgânico foram realizados por Rodrigues et al. (2010) com mudas de tomateiro e Costa et al. (2010 b) com muda de pepineiro.

Trabalhos com formação de mudas de tamarindeiro foram desenvolvidos por Mendonça et al. (2008), que avaliaram substrato (terra, esterco de curral curtido e areia lavada, na proporção de 3:2:1(v/v), adubado com as doses de $0 ; 3 ; 6$ e $9 \mathrm{~kg}$ de Osmocote ${ }^{\circledR}$ por $\mathrm{m}^{-3}$ de substrato) e ambientes (estufa agrícola, telado e céu aberto), por Silva et al. (2007) com o crescimento de mudas transplantadas a campo, por Flores et al. (2009) com a emergência e crescimento inicial, por Almeida et al. (2010) com a massa da semente e profundidade de semeadura, por Paula et al. (2009) com doses de sulfato de potássio na produção de porta-enxerto, por Queiroz et al. (2011) com a emergência e crescimento inicial em diferentes substratos (solo; solo e esterco de galinha; solo e esterco bovino; solo e esterco caprino; solo e terra vegetal, na proporção de 2:1), por Oliveira e Morais (1999) na remoção de cotilédones para o desenvolvimento de ramificações nas axilas cotiledonares e por Góes (2011) com enxertia, na qual os melhores métodos são garfagem no topo em fenda cheia, garfagem no topo à inglesa complicada e garfagem no topo à inglesa simples. Segundo Pereira et al. (2007) plantas produzidas por enxertia apresentam maior produção.

Este trabalho teve como objetivo avaliar a formação de mudas de pé-franco de tamarindeiro em diferentes composições de substratos e ambientes protegidos, na região de Aquidauana - MS. 


\section{MATERIAL E MÉTODOS}

Os experimentos com formação de mudas de pé -franco de tamarindeiro (Tamarindus indica L.) foramconduzidos na Universidade Estadual de Mato Grosso do Sul, Unidade Universitária de Aquidauana, que se localiza a altitude de $174 \mathrm{~m}$, longitude de $55^{\circ}$ $67^{\prime} \mathrm{W}$ e latitude de $20^{\circ} 45^{\prime} \mathrm{S}$, de novembro de 2009 a fevereiro de 2010. O clima da região, de acordo com a classificação de Köppen, é Aw, definido como clima tropical úmido e com temperatura média anual de $29^{\circ} \mathrm{C}$.

O desenvolvimento das mudas de tamarindeiro foi avaliado em diferentes ambientes de cultivo protegido e substratos. Utilizaram-se três diferentes ambientes protegidos: (A1) estufa agrícola em arco $(8,00 \mathrm{~m} \times 18,00 \mathrm{~m} \times 4,00 \mathrm{~m})$ de estrutura em aço galvanizado, com abertura zenital na cumeeira, coberta com filme de polietileno de $150 \mu \mathrm{m}$, difusor de luz, possuindo tela termorrefletora de $50 \%$ sob o filme e fechamentos laterais e frontais com tela de monofilamento, malha para 50\% de sombra; (A2) viveiro agrícola telado, de estrutura em aço galvanizado $(8,00$ $\mathrm{m} \times 18,00 \mathrm{~m} \times 3,50 \mathrm{~m}$ ), fechamento em $45^{\circ}$ de inclinação, com tela de monofilamento $\left(\right.$ Sombrite $\left.^{\circledR}\right)$, malha com $50 \%$ de sombra e (A3) viveiro agrícola telado de estrutura em aço galvanizado $(8,00 \mathrm{~m}$ x $18,00 \mathrm{~m} \mathrm{x}$ $3,50 \mathrm{~m}$ ), fechamento em $45^{\circ}$ de inclinação, com tela termorrefletora com $50 \%$ de sombra (Aluminet ${ }^{\mathbb{R}}$ ).

Em cada ambiente de cultivo, foram testados substratos à base de composto orgânico $(\mathrm{CO})$ e solo (SL): $100 \% \mathrm{SL}(\mathrm{S} 1) ; 80 \% \mathrm{SL}+20 \% \mathrm{CO}$ (S2); 60\% $\mathrm{SL}+40 \% \mathrm{CO}(\mathrm{S} 3) ; 40 \% \mathrm{SL}+60 \% \mathrm{CO}(\mathrm{S} 4) ; 20 \%$ $\mathrm{SL}+80 \%$ CO (S5) e 100\% CO (S6). As mudas foram produzidas em sacos de polietileno de 15,0 $\mathrm{x} 21,50 \mathrm{~cm}$.

O efeito do (ambiente foi examinado?) usando procedimento estatístico para análise de experimentos combinados (grupos de experimentos). Dentro dos ambientes, um delineamento experimental inteiramente casualizado foi adotado, com oito repetições, tendo cada repetição a média de duas mudas. Para realizar este tipo de análise (grupos de experimentos), deve-se, inicialmente, avaliar os quadrados médios dos resíduos (QMR) dos experimentos nas análises de variâncias individuais, e a razão entre o maior e o menor QMR não deve ultrapassar 7 (BANZATTO; KRONKA, 2006). Foi utilizado o programa estatístico Sisvar 5.3, sendo as médias das interações (ambientes e substratos) comparadas pelo teste de Tukey, a $5 \%$ de probabilidade.

O solo, classificado como Argissolo Vermelho-Amarelo (EMBRAPA, 2006), foi misturado ao composto orgânico Organosuper ${ }^{\circledR}$ (material comer- cial, composto por resíduos de frigoríficos, bagaço de cana, frutas, legumes, cereais, entre outros). A metodologia de análise química dos substratos foi a seguinte: substratos com até $50 \%$ de composto orgânico foram analisados como solo. Substratos com mais de $50 \%$ de composto orgânico foram analisados como adubo orgânico (Tabela 1).

As sementes do tamarindeiro foram coletadas de plantas nativas, na região de Aquidauana - MS, no mês de dezembro. Foi realizada a despolpa dos frutos e separadas as sementes, sendo lavadas em água corrente e secadas à sombra por dois dias.

O preparo do substrato e o preenchimento dos recipientes foram realizados em novembro. Os substratos ficaram em repouso nos sacos de polietileno por 14 dias, antes da realização da semeadura, dentro dos ambientes protegidos, para melhor mineralização da matéria orgânica, sendo irrigados todos os dias manualmente Durante a condução dos experimentos, a irrigação foi manual com regador, duas vezes ao dia, nos períodos matutino e vespertino, quando necessária, até a saturação do substrato, observada pelo início de escoamento de água. Nos viveiros telados (A2 e A3), em dias de chuva, os tratamentos não foram irrigados.

A precipitação nos meses de novembro, dezembro, janeiro e fevereiro foi, respectivamente, de 91,$2 ; 324,2 ; 304,0$ e $83,2 \mathrm{~mm}$.

As sementes foram embebidas em água por 24 horas; após esse tratamento, a semeadura foi realizada em 8 de dezembro de 2009, sendo foram semeadas duas sementes por recipiente, a uma profundidade de $2 \mathrm{~cm}$ e, quando estas estavam com folhas definitivas, realizou- se o desbaste. Após a semeadura e início da emergência das mudas, iniciou-se a avaliação do índice de velocidade de emergência (IVE) e da porcentagem de emergência (PE). Os dados foram coletados diariamente, do dia 15 a 27 de dezembro de 2009, até a estabilidade de um dos tratamentos, ou seja, quando a contagem nas últimas três datas não variou para o tratamento com maior número de plantas emergidas.

Foram realizadas mensurações de altura das plantas (AP), número de folhas (NF), diâmetro do colo (DC) e as fitomassas das matérias secas da parte aérea (MSPA) e do sistema radicular (MSSR). Somaram-se as MSPAs e MSSRs para obtenção de fitomassa da matéria seca total (MST). Foram determinadas as razões altura,diâmetro do colo-1 (RAD) e índice de qualidade de Dickson (IQD).

O índice de qualidade de Dickson (IQD) é uma fórmula balanceada, em que se incluem as relações das variáveis morfológicas, como MST, MSPA, MSSR, AP e DC (CRUZ et al., 2006), des- 
crito a seguir:

$$
I Q D=\frac{M S T(\mathrm{~g})}{\frac{A P(\mathrm{~cm})}{D C(\mathrm{~mm})}+\frac{\operatorname{MSPA}(\mathrm{g})}{\operatorname{MSSR}(\mathrm{g})}}
$$

Foram mensuradas diariamente as temperaturas de bulbo seco e bulbo úmido, às 09, 12e 15em cada ambiente de cultivo, durante a condução dos experimentos. Posteriormente, foi determinada a umidade relativa com auxílio do software Psychrometric Function Demo (Tabela 2).

\section{RESULTADOS E DISCUSSÃO}

Para todas as variáveis morfológicas e suas relações, a relação entre os quadrados médios do resíduo (RQMR) das análises de variâncias individuais dos experimentos não ultrapassou a relação de 7:1 (Tabela 3), permitindo, portanto, a realização da análise conjunta dos experimentos (grupos de experimentos). Para todas as variáveis estudadas, as interações apresentaram diferenças significativas.

Estes resultados demonstram que, para as mudas de tamarindeiro, existe influência entre o tipo de substrato e do ambiente protegido utilizado na germinação das sementes, na emergência das mudas e, consequentemente, na formação e no desenvolvimento das mudas, e estes fatores interagem entre si para proporcionar o melhor tipo de ambiente-substrato no crescimento da plântula. Interações entre substratos com "Organosuper ${ }^{\mathbb{B}}+$ Solo" e ambientes protegidos foram observadas em mudas de frutíferas por Santos et al. (2011) com o jatobazeiro-do-cerrado, Costa et al. (2010 a) com maracujazeiro e Costa et al. (2011) com o mamoeiro.

Para as variáveis de emergência do tamarindeiro, verificou-se, na avaliação dos substratos dentro de cada ambiente de cultivo, que nas mudas da estufa agrícola (A1) não foram observadas diferenças nos substratos avaliados (Tabela 4). Nos viveiros com telas (A2 e A3), quanto maior a porcentagem do composto no substrato maior foi o índice de velocidade de emergência das mudas (Tabela 4). Devido à maior hidratação da semente e seu intumescimento, nos viveiros telados, ocorreram maior velocidade de emergência, em função do aumento do volume, e a protrusão radicular mais rapidamente, conforme destacado por Sousa et al. (2010). Segundo Canellas et al. (2000) e Dalmago et al. (2009), quanto maior o teor de matéria orgânica maior a porosidade total. Desta forma, nos viveiros telados, os substratos com maior quantidade de matéria orgânica promoveram maior porosidade, favoreceram a aeração e propi- ciaram às mudas maior IVE.

Devido às malhas dos ambientes $\mathrm{A} 2$ e $\mathrm{A} 3$ propiciar infiltração de água pluvial, houve valores baixos do IVE nas mudas dos substratos contendo maior porcentagem de solo (Tabela 4).

$\mathrm{Na}$ estufa agrícola (A1), não foram observadas diferenças na porcentagem de emergência das mudas nos diversos substratos estudados. No entanto, nos viveiros telados (A2 e A3), as mudas do substrato $100 \%$ SL apresentaram menores porcentagens que as mudas cultivadas nos substratos com elevado teor de matéria orgânica $(60 ; 80$ e 100\% CO) (Tabela 4). Flores et al. (2009), que trabalharam com formação de mudas de tamarindeiro para fins agroflorestais utilizando sementes recém-colhidas ou com no máximo um mês de armazenamento, ambas semeadas com o hilo voltado para baixo, obtiveram alta porcentagem de emergência ( 97,5 e 96,5\%, respectivamente), depois de 30 dias.

Nos substratos com elevado teor de $\mathrm{CO}$, a partir de $60 \%$, os ambientes de cultivo não apresentaram diferenças para a PE do tamarindeiro; no entanto, nos substratos com menor $\mathrm{CO}$, inferior a $60 \%$, a estufa agrícola propiciou maior $\mathrm{PE}$ que os viveiros telados (Tabela 4). Nos substratos com menores porcentagens de $\mathrm{CO}$, a cobertura de polietileno propiciou melhores condições ambientais que os viveiros telados, permitindo maior porcentagem de plantas emergidas.

A cobertura por filme de polietileno propiciou às mudas dos substratos, com menor $\mathrm{CO}$, maiores velocidades de emergência devido à melhor condição do ambiente, pois o filme armazena maior quantidade de energia térmica no interior da estufa (CUNHA; ESCOBEDO, 2003). Segundo Sousa et al. (2010), as sementes de tamarindo germinam a partir dos 10 DAS, podendo chegar à sua completa emergência em até um mês. No presente estudo, o valor do início da emergência (7,0 dias), encontrado em estufa agrícola, mostra que este ambiente favoreceu a germinação das sementes.

Foram observadas altura de $43,4 \mathrm{~cm}$ na estufa agrícola e 40,3 cm no telado de monofilamento (Tabela 4), evidenciando maiores mudas em ambientes cobertos com polietileno de baixa densidade (PEBD), semelhante ao observado por Mendonça et al. (2008), que encontraram, aos $150 \mathrm{DAS}$, altura de $60 \mathrm{~cm}$ na estufa e $40 \mathrm{~cm}$ em viveiro de monofilamento. Almeida et al. (2010) encontraram mudas com altura média de 40,21 cm aos 160 DAS, em telado de tela antiafídio.

As mudas atingiram porte (altura) similar às observadas por Pereira et al. (2010), que trabalharam com diferentes recipientes e substratos (esterco de gado, camadefrango, húmus de minhoca, substrato artificial Plantmax numa relação composto orgânico/ 
terra de subsolo de 2/10), na produção de mudas de tamarindeiro, encontrando mudas com altura média de $42,9 \mathrm{~cm}$, em viveiro de monofilamento, aos 180 dias.

No telado de tela termorrefletora, foram observadas maiores mudas no substrato com $100 \%$ de CO. Este ambiente, que também permite a entrada de água pluvial, favoreceu a decomposição da matéria orgânica do composto, liberando nutrientes para a planta. Rodrigues et al. (2010), testando doses de 7; 14 e $21 \%$ desse composto orgânico, na formação de mudas de tomateiro, recomendaram a menor dose. Por ser uma planta mais rústica e de porte maior, o tamarindeiro desenvolveu- se bem em porcentagens maiores desse composto no presente trabalho.

$\mathrm{Na}$ análise do número de folhas (NF) no interior da estufa agrícola (A1), foi observado que as mudas dos substratos com $80 \% \mathrm{SL}+20 \% \mathrm{CO}$ e $40 \% \mathrm{SL}+60 \% \mathrm{CO}$ apresentaram número de folhas elevado, resultado também observado no telado de monofilamento (A2). No telado de tela termorrefletora, esta observação foi verificada nos substratos com $80 \%$ SL $+20 \%$ CO e $100 \%$ CO (Tabela 4 ). Assim como observado para a altura de planta em todos os substratos, o número de folhas das mudas da estufa agrícola foi mais expressivo que o dos demais ambientes.

No presente trabalho, o diâmetro de colo, em todos os ambientes de cultivo e nos substratos com composto orgânico a partir de $20 \%$ (Tabela 5), as mudas apresentaram menores diâmetros que as obtidas por Pereira et al. (2010), em substrato com composto orgânico e solo, no interior de viveiro de monofilamento, pois estas foram avaliadas aos 180 DAS, assim como por Almeida et al. (2010) aos 160 DAS.

As menores fitomassas secas da parte aérea e total foram observadas nas mudas oriundas do substrato solo (Tabela 5), diferentemente do encontrado por Santos et al. (2011), em mudas do jatobá-do-cerrado, que verificaram melhor produção de fitomassa seca da parte aérea em substratos contendo $100 \%$ de solo, quando cultivadas em estufa agrícola, comparadas às cultivadas em viveiros telados. Pereira et al. (2010), aos 180 DAS, encontraram fitomassa seca da parte aérea em mudas de tamarindo de 4,2 $\mathrm{g}$ em viveiro de monofilamento, utilizando composição de substrato com composto orgânico e solo. No presente experimento, as fitomassas secas da parte aérea foram menores, porém ressalta-se que a presente avaliação ocorreu aos 75 dias (Tabela 5)

Santos et al. (2011) observaram que a utilização de 40; 50; 60; 70 e 80\% de Organosuper ${ }^{\circledR}$ no substrato com solo propiciou maior fitomassa seca total em plantas cultivadas no interior de viveiro telado, que as cultivadas em estufa. Esse resultado também foi verificado para mudas de tamarindeiro neste trabalho, o qual substrato com teor de matéria orgânica de $80 \%$, no interior do viveiro telado, propiciou plantas com maior fitomassa.

Em todos os ambientes, a formulação de $80 \% \mathrm{SL}+20 \% \mathrm{CO}$, assim como a formulação de $40 \% \mathrm{SL}+60 \%$ CO do telado de monofilamento e a de $100 \%$ CO da tela termorrefletora propiciaram mudas com maior sistema radicular (Tabela 5). Este viveiro de tela termorrefletora $\left(\right.$ Aluminet $^{\mathbb{R}}$ ) possuía a mesma característica do viveiro de monofilamento $\left(\right.$ Sombrite ${ }^{\mathbb{R}}$ ), ou seja, ambos permitiam a entrada de água de chuva. Com os elevados valores de chuvas ocorridas nos meses de dezembro e janeiro, essa maior quantidade pode ter favorecido o desenvolvimento radicular das mudas no substrato com $100 \%$ de $\mathrm{CO}$, eliminando possíveis excesso de nutrientes que estariam causando fitotoxidade às mudas. Mendonça et al. (2008), utilizando sacos de 2,5 L, encontraram fitomassa seca do sistema radicular de tamarindeiro de $2,91 \mathrm{~g}$, aplicando dose de 4,28 $\mathrm{kg} \cdot \mathrm{m}^{-3}$ de Osmocote ${ }^{\circledR}$, aos 150 DAS, valores bem superior aos observados no presente trabalho, aos 75 DAS, com sacos de 1,54 L (15,0 x 21,5 cm). Os recipientes com maior volume $(2,5 \mathrm{~L})$ permitiram maior desenvolvimento do sistema radicular que os de menor volume $(1,54 \mathrm{~L})$. Em viveiro de tela preta com 50\% de sombreamento, aos 180 DAS, Pereira et al. (2010) encontraram médias de 6,4 e 7,4 g para MSSR em plantas cultivadas no substrato composto por cama de frango, aos 180 DAS, com recipientes de 12,0 × 24,0 cm e 18,0 × 30,0 cm, respectivamente.

Os resultados da fitomassa seca total das mudas seguiram a mesma tendência dos resultados obtidos parasa fitomassa seca aérea, com maiores valores nas mudas cultivadas em estufa agrícola, para a maioria dos substratos, excetuando apenas aqueles com $100 \%$ CO (Tabela 5). Para a fitomassa seca total de mudas de tamarindeiro, Mendonça et al. (2008) observaram que, em ambiente a céu aberto e fertilizante Osmocote ${ }^{\circledR}$, doses de $6,80 \mathrm{~kg} \cdot \mathrm{m}^{-3}$ promoveram melhor fitomassa seca $(13,56 \mathrm{~g})$ aos 150 DAS, em sacos de polietileno de 2,5 L.

Pelo observado nas Tabelas 4 e 5, verifica-se, tanto na emergência quanto no crescimento, número de folhas e fitomassas do tamarindeiro, que o filme de polietileno propiciou melhores condições internas de ambiente para a formação das mudas de tamarindeiro.

O valor resultante da divisão da altura da parte aérea de uma muda pelo respectivo diâmetro do colo (RAD) demonstra um equilíbrio de crescimento e pode demonstrar se houve estiolamento da 
muda. As mudas do substrato com $80 \% \mathrm{SL}+20 \%$ $\mathrm{CO}$ na estufa e no viveiro de monofilamento, assim como as mudas do substrato com $40 \% \mathrm{SL}+60 \%$ $\mathrm{CO}$ no viveiro de monofilamento e as com $100 \% \mathrm{CO}$ no viveiro de termorrefletora apresentaram maiores RADs, no entanto não podem ser consideradas mudas que estiolaram, pois foram as que apresentaram maiores alturas, maiores diâmetros e fitomassas (Tabela 5). Pereira et al. (2010) observaram valores de $\operatorname{RAD}\left(10,62 \mathrm{~cm} \cdot \mathrm{mm}^{-1}\right)$, em mudas de tamarindeiro, superiores às obtidas neste trabalho, em Aquidauana-MS. Nos três ambientes, o substrato S1 (100\% SL) apresentou plantas com os menores valores de RAD (Tabela 5), inferindo que elas apresentariam maior capacidade de resistência a campo, contudo tiveram as menores alturas e os menores diâmetros. Esse índice, para o tamarindeiro, não expressou a qualidade das mudas.

$\mathrm{Na}$ estufa agrícola, as mudas do substrato com $80 \% \mathrm{SL}+20 \% \mathrm{CO}$ apresentaram maior IQD $(0,35)$, enquanto no viveiro de monofilamento, foram as mudas dos substratos com porcentagens de composto orgânico a partir de $60 \%(0,22)$ e no viveiro de termorrefletora as com $100 \% \mathrm{CO}(0,26)$ (Tabela 5). Os valores encontrados para o IQD, neste trabalho, variaram de 0,18 a 0,35 . Pelo IQD, assim como em outras variáveis, os substratos com menores porcentagens de matéria orgânica apresentaram mudas com maior qualidade na estufa agrícola (Tabela 5). Os viveiros telados permitiram a utilização de maior quantidade de composto orgânico no substrato, como pode ser observado pelo IQD das mudas dos substratos com 60;80 e 100\% de CO.

Os teores de $\mathrm{Zn}$ (zinco) e S (enxofre) aumentaram extraordinariamente com o aumento das porcentagens do composto. Por exemplo, o enxofre (S) passou de 4,20 mg/dm para $3.090 \mathrm{mg} / \mathrm{dm}^{3}$ do $\mathrm{S} 1$ para o S6. Semelhança também verificada para o zinco
(Zn), que passou de $2,32 \mathrm{mg} / \mathrm{dm}^{3}$ para $26,2 \mathrm{mg} / \mathrm{dm}^{3}$ (Tabela 1). Altos valores de $\mathrm{Zn}$ causaram fitotoxidade em mudas do jatobá (Hymenaea courbaril L. var. stilbocarpa (Hayne) (DUBOC et al., 1996). Os valores altos de $\mathrm{Zn}$ podem ter caudado toxidade às mudas de tamarindeiro cultivadas nos substratos com elevada porcentagem deste composto orgânico no interior da estufa agrícola, , semelhantemente ao observado para mudas de Hymenaea stigonocarpa por Santos et al. (2011).

O cálcio $(\mathrm{Ca})$ é um elemento muito absorvido nos tecidos jovens (TAIZ; ZEIGER, 2009) e nos substratos com composto orgânico, e seus valores estavam elevados (Tabela 1). Seu excesso, muitas vezes, inibe a absorção de outros nutrientes, principalmente do $\mathrm{Mg}$ (DUBOC et al., 1996) e desta forma pode ter prejudicado o desenvolvimento das mudas de tamarindeiro.

Segundo Gomes et al. (2008), em mudas de Apuleia leiocarpa (garapa), espécie arbórea da família Fabaceae - Caesalpinoideae, os níveis de fósforo (P) no solo recomendado foi de $54 \mathrm{mg} \mathrm{dm}^{-3}$ e 191 $\mathrm{mg} \mathrm{dm}{ }^{-3}$, para saturação por bases de $60 \%$ e $40 \%$, respectivamente, com a obtenção da maior produção de matéria seca da parte aérea com a dose de $54 \mathrm{mg}$ $\mathrm{dm}^{-3}$,aos 120 dias. Para mudas de Anadenanthera colubrina (angico-branco), espécie arbórea da família Leguminosae - Mimosoideae, as doses de P de 127 e $126 \mathrm{mg} \mathrm{dm}^{-3}$, com saturação por base de 40 e $60 \%$, respectivamente, proporcionaram $90 \%$ da produção máxima após 170 dias de crescimento (GOMES et al., 2004). Fazendo uma analogia com o presente trabalho, a dose de $\mathrm{P}$ de $10,9 \mathrm{mg} \mathrm{dm}^{-3}$ explicou o menor crescimento das mudas do tamarindeiro no solo.

As doses dos nutrientes são importantes para o desenvolvimento das mudas, pois tanto o excesso como a falta causam desequilíbrios na absorção. As doses do composto orgânico elevou o pH, e as elevadas doses podem ter causado desbalanceamento nutricional e/ou efeito fitotóxico às mudas do tamarindeiro.

TABELA 1- Análise química dos substratos utilizados no experimento do tamarindeiro. Aquidauana, 08 de dezembro de 2009 a 20 de fevereiro de 2010.

\begin{tabular}{|c|c|c|c|c|c|c|c|c|c|c|c|c|c|}
\hline & ${ }^{(1)} \mathrm{pH}$ & $\mathrm{Ca}$ & $\mathrm{Mg}$ & $\mathrm{K}$ & $\mathrm{C}$ & $\mathrm{MO}$ & $\mathrm{P}$ & $\mathrm{Fe}$ & $\mathrm{Mn}$ & $\mathrm{Cu}$ & $\mathrm{Zn}$ & $\mathrm{B}$ & $\mathrm{S}$ \\
\hline$*$ & & \multicolumn{3}{|c|}{$\mathrm{Cmol}_{\mathrm{c}} \cdot \mathrm{dm}^{-3}$} & \multicolumn{2}{|c|}{ g.dm ${ }^{-3}$} & \multicolumn{7}{|c|}{ mg.dm ${ }^{-3}$} \\
\hline 'S1 & 5,3 & 2,5 & 0,7 & 0,1 & 7,8 & 13,5 & 10,9 & 9,1 & 51,9 & 0,1 & 2,3 & 0,9 & 4,2 \\
\hline${ }^{1} \mathrm{~S} 2$ & 6,7 & 6,1 & 4,2 & 0,9 & 32,9 & 56,7 & 499,8 & 86,3 & 174,4 & 1,4 & 31,2 & 1,6 & 528,0 \\
\hline${ }^{1} \mathrm{~S} 3$ & 6,9 & 7,3 & 5,6 & 1,1 & 51,2 & 88,0 & 544,8 & 89,2 & 165,5 & 1,8 & 31,5 & 2,6 & 726,0 \\
\hline${ }^{2} \mathrm{~S} 4$ & 6,7 & 13,2 & 10,7 & 3,5 & 92,5 & 159,1 & 642,4 & 127,8 & 171,7 & 3,6 & 26,1 & 11,4 & 1416,0 \\
\hline${ }^{2} \mathrm{~S} 5$ & 6,5 & 15,7 & 11,8 & 3,9 & 128,8 & 221,5 & 681,6 & 122,0 & 161,5 & 3,7 & 26,2 & 12,3 & 3030,0 \\
\hline${ }^{2} \mathrm{~S} 6$ & 6,7 & 14,9 & 11,9 & 3,9 & 139,8 & 240,4 & 704,8 & 118,7 & 147,9 & 4,0 & 26,2 & 15,9 & 3090,0 \\
\hline
\end{tabular}

$=$ Laboratório de análises: SOLANÁLISE, Dourados-MS.

${ }^{1}$ Extrator Melich: K - P - Fe - Mn - Cu e Zn; Extrator KCl: Ca - Mg - Al; Extrator HCl 0,05 N: B; Extrator Fosfato de Cálcio: S; ${ }^{2}$ Extrator $\mathrm{H}_{2} \mathrm{SO}_{4}$ : $\mathrm{P}$ - N; Extrator $\mathrm{HCl}$ : B; Extrator ácido nitroperclórico: $\mathrm{K}-\mathrm{Fe}-\mathrm{Mn}-\mathrm{Cu}-\mathrm{Zn}-\mathrm{S}-\mathrm{Ca}-\mathrm{Mg}-\mathrm{Al}$.

*(S1) $100 \%$ de solo; (S2) $80 \%$ de solo e $20 \%$ de composto orgânico; (S3) $60 \%$ de solo e $40 \%$ de composto orgânico; (S4) $40 \%$ de solo e $60 \%$ de composto orgânico; (S5) 20\% de solo e 80\% de composto orgânico; (S6) 100\% de composto orgânico. 
TABELA 2- Temperatura $\left({ }^{\circ} \mathrm{C}\right)$ e Umidade relativa (\%) média nos horários das $09 \mathrm{~h} 12 \mathrm{~h}$ e $15 \mathrm{~h}$ para cada ambiente (A) de cultivo, durante o experimento. Aquidauana, 08 de dezembro de 2009 a 20 de fevereiro de 2010.

\begin{tabular}{|c|c|c|c|c|c|c|c|c|c|}
\hline$*$ & TBS & TBU & TBS & TBU & TBS & TBU & & UR & \\
\hline & \multicolumn{2}{|c|}{ 09 Horas } & \multicolumn{2}{|c|}{12 Horas } & \multicolumn{2}{|c|}{15 Horas } & 09 Horas & 12 Horas & 15 Horas \\
\hline A1 & 28,6 & 25,1 & 31,9 & 26,1 & 32,5 & 26,2 & 75,9 & 65,1 & 63,2 \\
\hline A2 & 28,4 & 25,0 & 31,5 & 26,4 & 31,8 & 26,2 & 77,2 & 69,2 & 66,7 \\
\hline A3 & 28,9 & 26,0 & 32,2 & 27,5 & 32,3 & 27,3 & 79,8 & 70,1 & 68,4 \\
\hline EX & 28,5 & 25,3 & 31,4 & 26,5 & 32,3 & 26,6 & 78,8 & 69,8 & 66,7 \\
\hline
\end{tabular}

*TBS = Temperatura de bulbo seco $\left({ }^{\circ} \mathrm{C}\right) ; \mathrm{TBU}=$ Temperatura de bulbo úmido $\left({ }^{\circ} \mathrm{C}\right) ; \mathrm{UR}=$ Umidade relativa $(\%) ; \mathrm{A} 1$ = Estufa agrícola; $\mathrm{A} 2$ = Viveiro de monofilamento; $\mathrm{A} 3$ = Viveiro de termorrefletora; $\mathrm{EX}=$ Externa.

TABELA 3- Quadrado médio do resíduo das análises de variâncias individuais dos experimentos (ambientes) para o índice de velocidade de emergência (IVE), porcentagem de emergência (PE), altura de plantas (AP), número de folhas (NF), diâmetro do colo (DC), fitomassa seca da parte aérea (MSPA), fitomassa seca do sistema radicular (MSSR), fitomassa seca total (MST), razão altura e diâmetro (RAD) e índice de qualidade de Dickson (IQD) do tamarindeiro. Aquidauana MS, 2010.

\begin{tabular}{cccccc}
\hline \multicolumn{7}{c}{ IVE } & PE & AP & NF & DC \\
\hline \multicolumn{4}{c}{ Quadrado médio do resíduo das análises de variâncias individuais } \\
A1 & 0,211 & 395,833 & 1,151 & 2,504 & 0,014 \\
A2 & 0,172 & 311,198 & 1,042 & 2,603 & 0,022 \\
A3 & 0,177 & 395,833 & 5,216 & 3,188 & 0,022 \\
RQMR & 1,2 & 1,3 & 5,0 & 1,3 & 1,6 \\
CV(\%) & 30,56 & 24,54 & 4,80 & 7,94 & 3,97 \\
\hline & MSPA & MSSR & MST & RAD & IQD \\
\hline A1 & 0,026 & 0,0096 & 0,053 & 0,209 & 0,00043 \\
A2 & 0,013 & 0,0024 & 0,023 & 0,120 & 0,00028 \\
A3 & 0,033 & 0,0044 & 0,056 & 0,572 & 0,00041 \\
RQMR & 2,5 & 4,0 & 2,4 & 4,8 & 1,5 \\
CV(\%) & 9,87 & 14,00 & 7,51 & 5,97 & 6,89 \\
\hline
\end{tabular}

$\mathrm{CV}=$ Coeficiente de variação da análise conjunta; RQMR = Relação entre os quadrados médios dos resíduos máximos e mínimos dos diferentes ambientes de cultivo. A1 = Estufa agrícola; A2 = Viveiro de monofilamento; A3 = Viveiro de termorrefletora.

TABELA 4 -Interação entre ambientes e substratos (A x S) para o índice de velocidade de emergência (IVE), porcentagem de emergência (PE), altura de plantas (AP) e número de folhas (NF) do tamarindeiro. Aquidauana, 08 de dezembro de 2009 a 20 de fevereiro de 2010.

\begin{tabular}{ccccccc}
\hline$* *$ & $\mathrm{~A} 1$ & $\mathrm{~A} 2$ & $\mathrm{~A} 3$ & $\mathrm{~A} 1$ & $\mathrm{~A} 2$ & $\mathrm{~A} 3$ \\
\hline & $\mathrm{IVE}$ & & & $\mathrm{PE} \%)$ & \\
$100 \% \mathrm{SL}$ & $1,77 \mathrm{Aa} *$ & $0,28 \mathrm{Bb}$ & $0,41 \mathrm{Cb}$ & $75,0 \mathrm{Aa}$ & $31,3 \mathrm{Bb}$ & $50,0 \mathrm{Bab}$ \\
$80 \% \mathrm{SL}+20 \% \mathrm{CO}$ & $1,94 \mathrm{Aa}$ & $1,01 \mathrm{Bb}$ & $0,64 \mathrm{BCb}$ & $95,0 \mathrm{Aa}$ & $80,0 \mathrm{Aab}$ & $60,0 \mathrm{ABb}$ \\
$60 \% \mathrm{SL}+40 \% \mathrm{CO}$ & $1,76 \mathrm{Aa}$ & $0,85 \mathrm{Bb}$ & $0,81 \mathrm{BCb}$ & $90,0 \mathrm{Aa}$ & $55,0 \mathrm{ABb}$ & $65,0 \mathrm{ABab}$ \\
$40 \% \mathrm{SL}+60 \% \mathrm{CO}$ & $1,70 \mathrm{Aa}$ & $1,81 \mathrm{Aa}$ & $1,40 \mathrm{ABa}$ & $80,0 \mathrm{Aa}$ & $90,0 \mathrm{Aa}$ & $95,0 \mathrm{Aa}$ \\
$20 \% \mathrm{SL}+80 \% \mathrm{CO}$ & $1,77 \mathrm{Aa}$ & $1,87 \mathrm{Aa}$ & $1,70 \mathrm{Aa}$ & $90,0 \mathrm{Aa}$ & $85,0 \mathrm{Aa}$ & $90,0 \mathrm{Aa}$ \\
$100 \% \mathrm{CO}$ & $2,03 \mathrm{Aa}$ & $1,87 \mathrm{Aa}$ & $1,80 \mathrm{Aa}$ & $90,0 \mathrm{Aa}$ & $90,0 \mathrm{Aa}$ & $95,0 \mathrm{Aa}$ \\
\hline & & $\mathrm{AP}(\mathrm{cm})$ & & & $\mathrm{NF}$ & \\
$100 \% \mathrm{SL}$ & $30,1 \mathrm{Da}$ & $18,7 \mathrm{Db}$ & $18,4 \mathrm{~Eb}$ & $19,5 \mathrm{Ca}$ & $14,1 \mathrm{~Eb}$ & $13,3 \mathrm{Db}$ \\
$80 \% \mathrm{SL}+20 \% \mathrm{CO}$ & $43,4 \mathrm{Aa}$ & $39,4 \mathrm{Ab}$ & $33,6 \mathrm{Bc}$ & $27,2 \mathrm{Aa}$ & $26,1 \mathrm{Aa}$ & $23,0 \mathrm{Ab}$ \\
$60 \% \mathrm{SL}+40 \% \mathrm{CO}$ & $32,8 \mathrm{Ca}$ & $26,8 \mathrm{Cb}$ & $26,6 \mathrm{Db}$ & $20,6 \mathrm{BCa}$ & $17,7 \mathrm{Db}$ & $16,7 \mathrm{Cb}$ \\
$40 \% \mathrm{SL}+60 \% \mathrm{CO}$ & $39,2 \mathrm{Ba}$ & $40,3 \mathrm{Aa}$ & $29,3 \mathrm{Cb}$ & $25,5 \mathrm{Aa}$ & $24,3 \mathrm{ABa}$ & $19,8 \mathrm{Bb}$ \\
$20 \% \mathrm{SL}+80 \% \mathrm{CO}$ & $39,2 \mathrm{Ba}$ & $32,1 \mathrm{Bb}$ & $31,3 \mathrm{Cb}$ & $22,8 \mathrm{Ba}$ & $20,1 \mathrm{CDb}$ & $19,8 \mathrm{Bb}$ \\
$100 \% \mathrm{CO}$ & $38,3 \mathrm{Ba}$ & $30,6 \mathrm{Bb}$ & $39,2 \mathrm{Aa}$ & $22,3 \mathrm{Ba}$ & $21,9 \mathrm{BCa}$ & $22,7 \mathrm{Aa}$ \\
\hline
\end{tabular}

* Letras iguais maiúsculas nas colunas para comparação dos substratos e letras minúsculas nas linhas para comparação dos ambientes

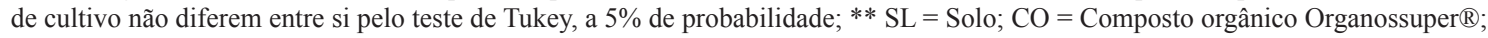
A1 = Estufa agrícola; A2 = Viveiro de monofilamento; A3 = Viveiro de termorrefletora . 
TABELA 5 - Interações entre ambientes e substratos (A x S) para o diâmetro do colo (DC), fitomassa seca total (MST), fitomassa seca da parte aérea (MSPA), fitomassa seca do sistema radicular (MSSR), razão altura e diâmetro do colo (RAD) e índice de qualidade de Dickson (IQD) do tamarindeiro, aos 75 DAS. Aquidauana - MS, 08 de dezembro de 2009 a 20 de fevereiro de 2010.

\begin{tabular}{ccccccc}
\hline & $\mathrm{A} 1$ & $\mathrm{~A} 2$ & $\mathrm{~A} 3$ & $\mathrm{~A} 1$ & $\mathrm{~A} 2$ & $\mathrm{~A} 3$ \\
\hline & \multicolumn{3}{c}{$\mathrm{DC}(\mathrm{mm})$} & & $\mathrm{MST}(\mathrm{g})$ & \\
$100 \% \mathrm{SL}$ & $3,46 \mathrm{Ca}$ & $2,66 \mathrm{Db}$ & $2,77 \mathrm{Db}$ & $3,15 \mathrm{BCa}$ & $1,41 \mathrm{Db}$ & $1,35 \mathrm{~Eb}$ \\
$80 \% \mathrm{SL}+20 \% \mathrm{CO}$ & $4,13 \mathrm{ABa}$ & $3,66 \mathrm{Bb}$ & $3,55 \mathrm{ABb}$ & $4,52 \mathrm{Aa}$ & $3,43 \mathrm{Ab}$ & $3,07 \mathrm{Bc}$ \\
$60 \% \mathrm{SL}+40 \% \mathrm{CO}$ & $3,58 \mathrm{Ca}$ & $3,21 \mathrm{Cb}$ & $2,84 \mathrm{Dc}$ & $2,62 \mathrm{Da}$ & $1,83 \mathrm{Cb}$ & $1,70 \mathrm{Db}$ \\
$40 \% \mathrm{SL}+60 \% \mathrm{CO}$ & $4,19 \mathrm{Aa}$ & $3,99 \mathrm{Ab}$ & $3,28 \mathrm{Cc}$ & $3,26 \mathrm{Ba}$ & $3,47 \mathrm{Aa}$ & $2,35 \mathrm{Cb}$ \\
$20 \% \mathrm{SL}+80 \% \mathrm{CO}$ & $4,06 \mathrm{ABa}$ & $3,66 \mathrm{Bb}$ & $3,40 \mathrm{BCc}$ & $2,92 \mathrm{Ca}$ & $2,85 \mathrm{Ba}$ & $2,49 \mathrm{Cb}$ \\
$100 \% \mathrm{CO}$ & $3,97 \mathrm{Ba}$ & $3,36 \mathrm{Cc}$ & $3,74 \mathrm{Ab}$ & $3,27 \mathrm{Bb}$ & $2,86 \mathrm{Bc}$ & $3,69 \mathrm{Aa}$ \\
\hline \multicolumn{7}{c}{$\mathrm{MSPA}(\mathrm{g})$} \\
$100 \% \mathrm{SL}$ & $2,23 \mathrm{Ca}$ & $1,26 \mathrm{Db}$ & $1,11 \mathrm{~Eb}$ & $0,91 \mathrm{Ba}$ & $0,15 \mathrm{Cc}$ & $0,24 \mathrm{Db}$ \\
$80 \% \mathrm{SL}+20 \% \mathrm{CO}$ & $3,25 \mathrm{Aa}$ & $3,07 \mathrm{Ab}$ & $2,52 \mathrm{Bc}$ & $1,27 \mathrm{Aa}$ & $0,37 \mathrm{Bc}$ & $0,55 \mathrm{Bb}$ \\
$60 \% \mathrm{SL}+40 \% \mathrm{CO}$ & $1,92 \mathrm{Da}$ & $1,68 \mathrm{Ca}$ & $1,46 \mathrm{Da}$ & $0,70 \mathrm{Ca}$ & $0,16 \mathrm{Cb}$ & $0,24 \mathrm{Db}$ \\
$40 \% \mathrm{SL}+60 \% \mathrm{CO}$ & $2,73 \mathrm{Bb}$ & $2,96 \mathrm{Aa}$ & $1,93 \mathrm{Cc}$ & $0,53 \mathrm{Da}$ & $0,51 \mathrm{Aa}$ & $0,42 \mathrm{Cb}$ \\
$20 \% \mathrm{SL}+80 \% \mathrm{CO}$ & $2,54 \mathrm{Ba}$ & $2,24 \mathrm{Bb}$ & $1,98 \mathrm{Cc}$ & $0,39 \mathrm{Ec}$ & $0,61 \mathrm{Aa}$ & $0,51 \mathrm{BCb}$ \\
$100 \% \mathrm{CO}$ & $2,70 \mathrm{Bb}$ & $2,27 \mathrm{Bc}$ & $2,90 \mathrm{Aa}$ & $0,57 \mathrm{Db}$ & $0,60 \mathrm{Ab}$ & $0,79 \mathrm{Aa}$ \\
\hline \multicolumn{7}{c}{$\mathrm{RAD}(\mathrm{cm} \cdot \mathrm{mm}-1)$} \\
$100 \% \mathrm{SL}$ & $8,69 \mathrm{Ca}$ & $7,04 \mathrm{Cb}$ & $6,60 \mathrm{Cb}$ & $0,28 \mathrm{Ba}$ & $0,09 \mathrm{Cc}$ & $0,12 \mathrm{Db}$ \\
$80 \% \mathrm{SL}+20 \% \mathrm{CO}$ & $10,52 \mathrm{Aa}$ & $10,75 \mathrm{Aa}$ & $9,47 \mathrm{Bb}$ & $0,35 \mathrm{Aa}$ & $0,18 \mathrm{Bc}$ & $0,22 \mathrm{Bb}$ \\
$60 \% \mathrm{SL}+40 \% \mathrm{CO}$ & $9,19 \mathrm{BCa}$ & $8,38 \mathrm{Bb}$ & $9,38 \mathrm{Ba}$ & $0,22 \mathrm{Ca}$ & $0,10 \mathrm{Cb}$ & $0,11 \mathrm{Db}$ \\
$40 \% \mathrm{SL}+60 \% \mathrm{CO}$ & $9,35 \mathrm{BCb}$ & $10,10 \mathrm{Aa}$ & $8,96 \mathrm{Bb}$ & $0,22 \mathrm{Ca}$ & $0,22 \mathrm{Aa}$ & $0,17 \mathrm{Cb}$ \\
$20 \% \mathrm{SL}+80 \% \mathrm{CO}$ & $9,66 \mathrm{Ba}$ & $8,76 \mathrm{Bb}$ & $9,22 \mathrm{Bab}$ & $0,18 \mathrm{Db}$ & $0,23 \mathrm{Aa}$ & $0,19 \mathrm{BCb}$ \\
$100 \% \mathrm{CO}$ & $9,67 \mathrm{Bb}$ & $9,11 \mathrm{Bb}$ & $10,48 \mathrm{Aa}$ & $0,23 \mathrm{Cb}$ & $0,22 \mathrm{Ab}$ & $0,26 \mathrm{Aa}$
\end{tabular}

* Letras iguais maiúsculas nas colunas para comparação dos substratos e letras minúsculas nas linhas para comparação dos ambientes

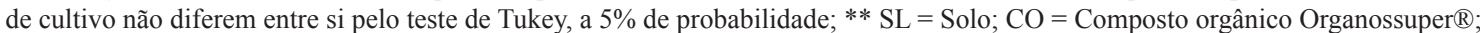
A1 = Estufa agrícola; A2 = Viveiro de monofilamento; A3 = Viveiro de termorrefletora.

\section{CONCLUSÕES}

1-A estufa agrícola é o melhor ambiente para a formação das mudas de tamarindeiro.

2-O substrato com $80 \%$ de solo e $20 \%$ de Organosuper $^{\circledR}$ é o mais propício para a formação das mudas, nos três ambientes, principalmente na estufa agrícola.

3-No telado de cor preta, pode-se utilizar o substrato com $60 \%$ de composto orgânico, e no telado aluminizado,com $100 \%$.

\section{REFERÊNCIAS}

ALMEIDA, M. S.; MELO, B.; SILVA, C. A.; SANTANA, D. G.; SILVA, C. J. Massa de sementes e profundidades de semeadura no desenvolvimento de mudas de tamarindeiro. Revista Brasileira de Fruticultura, Jaboticabal, v. 32, n. 2, p. 555-560, 2010.
BANZATTO, D. A.; KRONKA, S. N. Experimentação agrícola. 4.ed. Jaboticabal: FUNEP/UNESP, 2006. 237 p.

CANELLAS, L. P.; BERNER, P. G.; SILVA, S. H.; SILVA, M. B.; SANTOS, G. A. Frações da matéria orgânica em seis solos de uma topossequência no Estado do Rio de Janeiro. Pesquisa Agropecuária Brasileira, Brasília, v. 35, n. 1, p. 133-143, 2000.

COSTA, E.; LEAL, P. A. M.; GOMES, V. A.; MACHADO, D.; JARA, M. C. S. Biomassa de mudas de pepinos híbridos conduzidos sob ambientes protegidos. Bragantia, Campinas, v. 69, n. 2, p. 381-386, 2010 b.

COSTA, E.; LEAL, P. A. M.; SASSAQUI, A. R.; GOMES, V. A. G. Doses de composto orgânico comercial na composição de substratos para a produção de mudas de maracujazeiro em diferentes tipos de cultivo protegido. Engenharia Agrícola, Jaboticabal, v. 30, n. 5, p. 776-787, 2010 a. 
COSTA, E.; LEAL, P. A. M.; MESQUITA, V. do A. G.; SASSAQUI, A. R. Efeitos do Organosuper ${ }^{\circledR}$ e do ambiente protegido na formação de mudas de mamoeiro. Engenharia Agrícola, Jaboticabal, v. 31, n. 1, p. 41-55, 2011.

CRUZ, C. A. F.; PAIVA, H. N. de; GUERRERO, C. R. A. Efeito da adubação nitrogenada na produção de mudas de sete-cascas (Samanea inopinata (harms) ducke). Revista Árvore. Viçosa, MG, v. 30, n. 4, p. 537-546, 2006.

CUNHA, A. R.; ESCOBEDO, J. F. Alterações micrometeorológicas causadas pela estufa plástica e seus efeitos no crescimento e produção da cultura de pimentão. Revista Brasileira de Agrometeorologia, Santa Maria, v.11, n.1, p.15-27, 2003.

DALMAGO, G. A.; BERGAMASCHI, H.; BERGONCI, J. I.; . KRÜGER, C. A. M. B; COMIRAN, F.; HECKLER, B. M. M. Retenção e disponibilidade de água às plantas, em solo sob plantio direto e preparo convencional. Revista Brasileira de Engenharia Agrícola e Ambiental, Campina Grande, v. 13, p. 855-864, 2009. Suplemento.

DUBOC, E.; VENTURIN, N.; VALE, F.R.; DAVIDE, A.C. Nutrição do jatobá (Hymenae courbaril L. var. Stilbocarba (Haene) Lee et lang). Cerne, Lavras, v. 2, n. 1, p. 138-152, 1996.

EMBRAPA - Empresa Brasileira de Pesquisa Agropecuária. Sistema brasileiro de classificação de solos. Brasília: Centro Nacional de Pesquisa de Solos, 2006. 305p.

FLORES, E.; MORATINOS, P.; RAMIREZ, M.; GARCIA, D. E. Evaluación de la emergencia y las características morfológicas iniciales de Tamarindus indica L. con fines agroforestales. Pastos y Forrajes, Venezuela, v. 32, n. 2, p. 1-1, 2009. Disponível em: $\leq \mathrm{http}$ ://scielo.sld.cu/scielo.php?script=sci arttext\& pid=S0864-03942009000200008>. Acesso em: 04 ago. 2011.

GÓES, G. B. de. Propagação do tamarindeiro (Tamarindus indica L.) e da pitombeira (Talisia esculenta Raldk) por enxertia. 2011. $73 \mathrm{f}$. Dissertação (Mestrado em Agronomia: Fitotecnia) Universidade Federal Rural do Semiárido, Mossoró, 2011.
GOMES, K. C. O.; PAIVA, H. N.; NEVES, J. C. L.; BARROS, N. F.; SILVA, S. R. Influência da saturação por bases e do fósforo no crescimento de mudas de angico-branco. Revista Árvore, Viçosa, MG, v. 28, n. 6, p. 785-792, 2004.

GOMES, K. C. O.; PAIVA, H. N.; NEVES, J. C. L.; BARROS, N. F.; SILVA, S. R. Crescimento de mudas de garapa em resposta à calagem e ao fósforo. Revista Árvore, Viçosa, MG, v. 32, n. 3, p. 387-394, 2008.

KOTHARI, V.; SESHADRI, S. In vitro antibacterial activity in seed extracts of Manilkara zapota, Anona squamosa, and Tamarindus indica. Biological Research, Santiago, v. 43, n. 2, p. 165-168, 2010. Disponível em: < http://www.scielo.cl/scielo. php?pid $=$ S0716-97602010000200003\&script $=$ sci $\underline{\text { arttext }}>$. Acesso em: 04 ago. 2011.

MENDONÇA, V.; ABREU, N. A. A.; SOUZA, H. A.; TEIXEIRA, G. A.; HAFLE, O. M.; RAMOS, J. R. Diferentes ambientes e Osmocote ${ }^{\circledR}$ na produção de mudas de tamarindeiro (Tamarindus indica). Ciência e Agrotecnologia, Lavras, v. 28, n. 2, p. 391-397, 2008.

OLIVEIRA, O. F.; MORAIS, P. L. D. Influência da remoção de cotilédones no desenvolvimento de ramificações nas axilas cotiledonares de plântulas de leguminosas. Acta Botanica Brasilica, Porto Alegre, v. 13, n. 3, p. 243-249, 1999.

PAULA, Y. C. M.; MENDONÇA, V.; GÓES, G. B.; LIMA, A. S.; MEDEIROS, L. F.; BATISTA, T. M. V. Doses de sulfato de potássio na produção de porta-enxerto de tamarindeiro (Tamarindus indica L.). Agrarian, Dourados, v. 2, n. 5, p. 71-79, 2009.

PEREIRA, P. C.; MELO, B.; FRAZÃO, A. A.; ALVES, P. R. B. A cultura do tamarindeiro (Tamarindus indica L.). 2007. Disponível em: http://www.fruticultura.iciag.ufu.br./tamarindo.html. Acesso em: 10 ago. 2012.

PEREIRA, P. C.; MELO, B.; FREITAS, R. S.; TOMAZ, M. A.; TEIXEIRA, I. R. Tamanho de recipientes e tipos de substrato na qualidade de mudas de tamarindeiro. Revista Verde de Agroecologia e Desenvolvimento Sustentável, Mossoró, v. 5, n. 3, p. 136-142, 2010. 
QUEIROZ, J. M. O.; DANTAS, A. C. V. L.; ALMEIDA, V. O.; BARROSO, J. P. Emergência de plântulas e crescimento inicial de tamarindeiro em diferentes substratos. Magistra, Cruz das Almas, v. 23, n. 4, p. 221-227, 2011.

RAO POPURI, S.; JAMMALA, A.; NAGA SURESH REDDY, K. V.;ABBURI, K. Biosorption of hexavalent chromium using tamarind (Tamarindus indica) fruit shell-a comparative study. Electronic Journal of Biotechnology, Valparaiso, v. 10, n. 3, p. 358-367, 2007. Disponível em: <http://www.scielo.cl/scielo. php?pid $=$ S0717-34582007000300003\&script $=$ sci arttext>. Acesso em: 04 ago 2011.

RODRIGUES, E. T.; LEAL, P. A. M.; COSTA, E.; PAULA, T. S.; GOMES, V. A. Produção de mudas de tomateiro em diferentes substratos e recipientes em ambiente protegido. Horticultura Brasileira, Brasília, v. 28, n. 4; p. 483-488, 2010.

SANTOS, L. C. R.; COSTA, E.; LEAL, P. A. M.; NARDELLI, E. M. V.; SOUZA, G. S. A. de. Ambientes protegidos e substratos com doses de composto orgânico comercial e solo na formação de mudas de jatobazeiro em Aquidauana-MS. Engenharia Agrícola, Jaboticabal, v. 31, n. 2, p. 249-259, 2011.
SILVA, P. S. L.; SILVA, K. M. B.; LÔBO, R. N. B.; SILVA, P. I. B. Growth of seven perennial plant species adapted to the Brazilian Semi-Arid. Acta Botanica Brasilica, São Paulo, v. 21, n. 4, p. 935941, 2007.

SOUSA, D. M. M.; BRUNO, R. L. A.; DORNELAS, C. S. M.; ALVES, E. U.; ANDRADE, A. P.; NASCIMENTO, L. C. Caracterização morfológica de frutos e sementes e desenvolvimento pósseminal de Tamarindus indica L. - Leguminosae: caesalpinioideae. Revista Árvore, Viçosa, MG, v. 34, n. 6, p. 1.009-1.015, 2010.

TAIZ, L.; ZEIGER, E. Fisiologia vegetal. 4.ed. Porto Alegre: Artmed, 2009. 719p.

YAMANISHI, O. K.; FAGUNDES, G. R.; MACHADO FILHO, J. A.; VALONE, G. V. Efeito de diferentes substratos e duas formas de adubação na produção de mudas de mamoeiro. Revista Brasileira de Fruticultura, Jaboticabal, v. 26, n. 2 , p. 276-279, 2004. 